\title{
CYCLIC SPECTRAL BASED APPROACH TO CLOSED-LOOP IDENTIFICATION
}

\author{
Lianming Sun* Akira Sano** \\ * Department of Information and Media Sciences \\ The University of Kitakyushu \\ 1-1 Hibikino, Wakamatsu-ku, Kitakyusyu, 808-0135 Japan \\ ** Department of System Design Engineering \\ Keio University \\ 3-14-1 Hiyoshi, Kohoku-ku, Yokohama, 223-8522 Japan
}

\begin{abstract}
The direct closed-loop identification algorithm based on cyclic spectral is considered in frequency domain in this paper. It is illustrated that the input and output signals obtained through inter-sampling technique have cyclic spectral, and the cyclic spectral contain plant model information, then the plant model can be estimated just from the cyclic spectral of the plant input and output signals. It is clarified that the test signal is not necessary, and the new algorithm does not depend on the controller structure. The effectiveness of the proposed algorithm is also demonstrated through several numerical examples. Copyright(c)2005 IFAC.
\end{abstract}

Keywords: Identification, closed-loop identification, multirate, frequency estimation

\section{INTRODUCTION}

Feedback control plays an important role in control engineering. Consequently closed-loop identification has attracted a great deal of reserchers' attention. Several closed-loop identification methods have been developed, and they can be classified into three main categories such as the direct methods (Ljung et al., 1974; Wellstead and Edmunds, 1975), the indirect methods (Hansen and Franklin, 1988; den Hof et al., 1995) and the joint input-output methods. In the indirect methods or the joint input-output methods, the external exciting test signals are used hence it is possible to reduce the closed-loop identification problem into several stable open loop identifications, where the external test signal is considered as the input signals of these open loops. Nevertheless, when the test signal is unavailable, or the power of test signal allowed in the regular operation is too small to do significant contribution to system identifica- tion, it seems that the direct method is the unique choice to the closed-loop identification problem. The identifiability condition for the conventional direct method usually requires that the order of the controller should be higher than that of the plant (Wellstead and Edmunds, 1975). However, under the case where the feedback controller is designed by using a reduced-order nominal model, it is hardly possible to satisfy the identifiability condition. It has been demonstrated that this problem can be treated by applying prediction error method in the time domain when the plant is stable (Sun et al., 2001), and by a subspace-based algorithm for an unstable plant (Sun et al., 1999) based on the output inter-sampling scheme.

On the other hand, only few frequency domain approaches have been published known as polyspectral or cyclic spectral (Tontiruttananon and Tugnait, 1998; Giannakis, 1995), which uses the external test signal with special probability density 
distribution, to deal with closed-loop identification. Nevertheless, it seems that there is not any frequency domain direct identification algorithm to give consistent plant model estimation from closed-loop data without using test signal.

In this paper we extend the output inter-sampling based approach into frequency domain whereas the closed-loop is performed without changing any regular operation conditions of feedback control. In the new approach, the plant output is sampled at a faster rate than the control input, so the intersampled input and output are cyclostationary in probability framework, hence they have cyclic spectral. It is clarified that the cyclic spectral expands the capability of identification in closedloop systems, even though the plants are unstable. It is also illustrated that the external test signal is not necessary, just the enough frequency excitation of the noise can ensure the possibility of closed-loop identification.

\section{PROBLEM STATEMENT}

Assume that the plant is stabilized through a discrete-time controller followed by a zero-order holder whose holding period is $T$, as shown in Fig 1. Thus the control input to the plant is a piecewise signal. Without loss of the generality, let the discrete-time $T$-model of the plant and controller be expressed by

$$
\begin{aligned}
& G\left(z^{-1}\right)=\frac{b_{1} z^{-1}+\cdots+b_{n} z^{-n}}{1+a_{1} z^{-1}+\cdots+a_{n} z^{-n}}:=\frac{B\left(z^{-1}\right)}{A\left(z^{-1}\right)} \\
& K\left(z^{-1}\right)=\frac{d_{0}+\cdots+d_{n_{K}} z^{-n_{K}}}{1+c_{1} z^{-1}+\cdots+c_{n_{K}} z^{-n_{K}}}:=\frac{D\left(z^{-1}\right)}{C\left(z^{-1}\right)}
\end{aligned}
$$

\subsection{System Description in Output Inter-Sampling}

Let the output sampling interval be denoted as $\Delta$ as shown in Fig 2, where $\Delta=T / p$, and $p$ is an integer. Then the closed-loop system can also be described by the model corresponding to sampling interval $\Delta$, where the plant model becomes to

$$
\begin{aligned}
G_{\Delta}\left(q^{-1}\right) & =\frac{b_{\Delta, 1} q^{-1}+\cdots+b_{\Delta, n} q^{-n}}{1+a_{\Delta, 1} q^{-1}+\cdots+a_{\Delta, n} q^{-n}} \\
& :=\frac{B_{\Delta}\left(q^{-1}\right)}{A_{\Delta}\left(q^{-1}\right)}
\end{aligned}
$$

Here $q^{-1}$ is a backward shift operator corresponding to the sampling interval $\Delta$, and $q^{-p}=z^{-1}$. Assume that the input-output relation of the plant is given $y_{\Delta}($ gr $)=G_{\Delta}\left(q^{-1}\right) u_{\Delta}(k)+e_{\Delta}(k)$

Let $y_{j}(m)$ and $e_{j}(m)$ be defined by $y_{j}(m)=$ $y_{\Delta}(m p+j)$ and $e_{j}(m)=e_{\Delta}(m p+j)$, respectively, where $0 \leq j<p$, then $y_{j}(m)$ is given by the following theorem (Sun et al., 2001).
Theorem 1. $y_{j}(m)$ can be described by the following multi-output model

$$
y_{j}(m)=G_{j}\left(z^{-1}\right) u(m)+e_{j}(m)
$$

whrer $G_{j}\left(z^{-1}\right)$ is given by

$$
\begin{aligned}
& G_{j}\left(z^{-1}\right)=\boldsymbol{c}^{T}\left(\boldsymbol{I}-\boldsymbol{A}^{p} z^{-1}\right) \\
& \quad\left(\sum_{i=0}^{j-1} \boldsymbol{A}^{i} \boldsymbol{b}+\sum_{i=j}^{p-1} \boldsymbol{A}^{i} \boldsymbol{b} z^{-1}\right)
\end{aligned}
$$

And $\boldsymbol{A}, \boldsymbol{b}$ and $\boldsymbol{c}$ are the state-space realization matrice of $G_{\Delta}\left(q^{-1}\right)$.

Corollary 2. The multi-output model $G_{j}\left(z^{-1}\right)$ shares common denominator polynomial with $G\left(z^{-1}\right)$. Furthermore, the poles of $G\left(z^{-1}\right)$ are the $p$-th power of the poles of $G_{\Delta}\left(q^{-1}\right)$.

Corollary 3. The parameters $b_{l}$ of numerical polynomial $B\left(z^{-1}\right)$ of $T$-model can be calculated by

$$
b_{l}=\sum_{l_{1}=(l-1) p+1}^{l p} \beta_{l_{1}}
$$

where $\beta_{l_{1}}$ are the coefficients of

$$
B_{\Delta}\left(q^{-1}\right) \bar{A}_{\Delta}\left(q^{-1}\right)=\beta_{1} q^{-1}+\beta_{2} q^{-2}+\cdots
$$

And $A_{\Delta}\left(q^{-1}\right)$ is a polynomial satisfying $A_{\Delta}\left(q^{-1}\right)$ $\bar{A}_{\Delta}\left(q^{-1}\right)=A\left(q^{-p}\right)$.

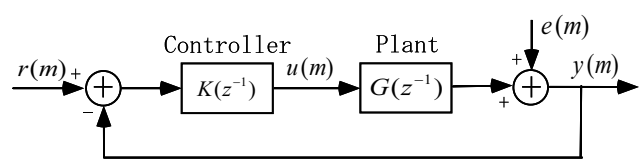

Fig. 1. Model corresponding to sampling period $T$

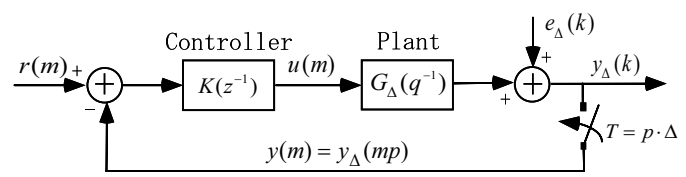

Fig. 2. Model corresponding to sampling period $\Delta$

\subsection{Signal Relations in Output Inter-Sampling}

Assume that the output noise $e_{\Delta}(k)$ is a stochastic process which is modeled by

$$
e_{\Delta}(k)=H_{\Delta}\left(q^{-1}\right) \varepsilon_{\Delta}(k)=\frac{F_{\Delta}\left(q^{-1}\right)}{E_{\Delta}\left(q^{-1}\right)} \varepsilon_{\Delta}(k)(9)
$$

where $\varepsilon_{\Delta}(k)$ is a white i.i.d noise with zero-mean and variance $\sigma_{\Delta}^{2}$. Following Theorem 1 , there is a polynomial such that

$$
E_{\Delta}\left(q^{-1}\right) \bar{E}_{\Delta}\left(q^{-1}\right)=E\left(q^{-p}\right)=E\left(z^{-1}\right)
$$

where $\bar{E}_{\Delta}\left(q^{-1}\right)=\prod_{j=1}^{p-1} E_{\Delta}\left(e^{-j i \frac{2 \pi}{p}} q^{-1}\right)$. Furthermore, let $F_{j}\left(q^{-p}\right)$ be the polynomial containing all the power $q^{-l p}$ of $q^{j} F_{\Delta}\left(q^{-1}\right) \bar{E}_{\Delta}\left(q^{-1}\right)$, then $e_{\Delta}(k)$ can be rewritten in

$$
e_{\Delta}(k)=\sum_{j=0}^{p-1}\left(\frac{F_{j}\left(z^{-1}\right)}{E\left(z^{-1}\right)} \varepsilon_{\Delta}(k-j)\right)
$$

where $F_{p}\left(z^{-1}\right)=z F_{0}\left(z^{-1}\right)$. Define $\varepsilon_{j}(m)=$ $\varepsilon_{\Delta}(m p+j)$, and let $\xi_{\Delta, j}(k)$ be a virtual signal by 
holding $\varepsilon_{j}(m)$ for $T$, then the signals with respect to $T$ and $\Delta$ respectively are expressed by

$$
\begin{aligned}
& w(m)=e(m)=\sum_{j=0}^{p-1}\left(\frac{F_{p-j}\left(z^{-1}\right)}{E\left(z^{-1}\right)} \varepsilon_{j}(m-1)\right) \\
& w_{\Delta}(k)=\sum_{j=0}^{p-1}\left(\frac{F_{p-j}\left(q^{-p}\right)}{E\left(q^{-p}\right)} \xi_{\Delta, j}(k)\right)
\end{aligned}
$$

For the simplicity of notation we assume that $r(m)=0$, then the control input to the plant with respect to $\Delta$ is formulated as

$$
u_{\Delta}(k)=\frac{-K\left(q^{-p}\right)}{1+K\left(q^{-p}\right) G\left(q^{-p}\right)} w_{\Delta}(k)
$$

\section{CYCLIC PROPERTY IN INTER-SAMPLING}

\subsection{Cyclic Property of Inter-Sampled Signals}

Define the correlation function $c_{x_{\Delta, 1} x_{\Delta, 2}}(k, \tau)$ as follows.

$$
c_{x_{\Delta, 1} x_{\Delta, 2}}(k, \tau):=E\left\{x_{\Delta, 1}(k+\tau) x_{\Delta, 2}(k)\right\}
$$

For example, the auto-correlation function $c_{\xi_{\Delta, j} \xi_{\Delta, j}}$ $(k, \tau)$ can be given by

$$
\begin{gathered}
c_{\xi_{\Delta, j} \xi_{\Delta, j}}(k, \tau)=E\left\{\xi_{\Delta, j}(k+\tau) \xi_{\Delta, j}(k)\right\} \\
\left\{\begin{array}{cc}
\sigma_{\Delta}^{2}, & \text { for } k=m p, \tau=0, \cdots, p-1 \\
\sigma_{\Delta}^{2}, & \text { for } k=m p+1, \tau=-1, \cdots, p-2 \\
\vdots \\
\sigma_{\Delta}^{2}, & \text { for } k=(m+1) p-1 \\
\tau=-p+1, \cdots, 0 \\
0, & \text { otherwise }
\end{array}\right.
\end{gathered}
$$

where $\sigma_{\Delta}^{2}$ is the variance of $\varepsilon_{\Delta}(k)$. The cross correlation function $c_{\xi_{\Delta, j} \varepsilon_{\Delta}}(k, \tau)$ is also given by

$$
\begin{aligned}
& c_{\xi_{\Delta, j} \varepsilon_{\Delta}}(k, \tau)=E\left\{\xi_{\Delta, j}(k+\tau) \varepsilon_{\Delta}(k)\right\} \\
= & \begin{cases}\sigma_{\Delta}^{2} & k=m p+j, \tau=0, \cdots, p-1 \\
0 & \text { otherwise }\end{cases}
\end{aligned}
$$

It can be claimed that the processes $x_{\Delta}(k)$ and $y_{\Delta}(k)$ are second-order cyclosationary processes since $c_{x_{\Delta} y_{\Delta}}(k, \tau)$ is a periodic function in $k$ (Tontiruttananon and Tugnait, 1998; Giannakis, 1995) when $x_{\Delta}(k)$ and $y_{\Delta}(k)$ are the sequences obtained through output inter-sampling. Next it will be shown that such processes have cyclic spectral.

Assume that the Fourier series of (15) with respect to $k$ exists, then the cyclic spectral and its Fourier transform can be defined by

$$
\begin{aligned}
& \mathcal{C}_{x_{\Delta} y_{\Delta}}(\alpha, \tau)=\lim _{N \rightarrow \infty} \frac{1}{N} \sum_{k=0}^{N-1} c_{x_{\Delta} y_{\Delta}}(k, \tau) e^{-i \alpha k} \\
& \mathcal{S}_{x_{\Delta} y_{\Delta}}(\alpha, \omega)=\sum_{\tau=-\infty}^{\infty} \mathcal{C}_{x_{\Delta} y_{\Delta}}(\alpha, \tau) e^{-i \omega \tau}
\end{aligned}
$$

where $\alpha \in \mathcal{A}$, and $\mathcal{A}$ is the cyclic angles set such that $\mathcal{A}=\left\{\alpha \mid \mathcal{C}_{x_{\Delta} y_{\Delta}}(\alpha, \tau) \neq 0,0 \leq \alpha<2 \pi(\bmod 2 \pi)\right\}$. It is clarified that $\mathcal{A}=\{j 2 \pi / p$, for $j=0,1, \cdots, p-1\}$ under output inter-sampling. For simplicity of notation, let $r_{\Delta}(k)=0$. Then the cyclic spectral of $\xi_{\Delta, j}(k), \varepsilon_{\Delta}(k)$ are summarized in following Lemma.

Lemma 4. The cross and auto-cyclic spectral of $\xi_{\Delta, j}(k)$ and $\varepsilon_{\Delta}(k)$ are given by

$$
\begin{aligned}
& \mathcal{S}_{\xi_{\Delta, j} \varepsilon_{\Delta}}(\alpha, \omega)=\sum_{\tau=-\infty}^{\infty} \mathcal{C}_{\xi_{\Delta, j} \varepsilon_{\Delta}}(\alpha, \tau) e^{-i \omega \tau} \\
= & e^{i j(\alpha-\omega)}\left(1+e^{-i \omega}+\cdots+e^{-i(p-1) \omega}\right) \mathcal{X} \\
& \mathcal{S}_{\xi_{\Delta, j} \xi_{\Delta, j}}(\alpha, \omega)=\sum_{\tau=-\infty}^{\infty} \mathcal{C}_{\xi_{\Delta, j} \xi_{\Delta, j}}(\alpha, \tau) e^{-i \omega \tau} \\
= & \left(1+e^{-i \omega}+\cdots+e^{-i(p-1) \omega}\right) \\
& \left(1+e^{-i(\alpha-\omega)}+\cdots+e^{-i(p-1)(\alpha-\omega)}\right) \mathcal{X}
\end{aligned}
$$

where $\mathcal{X}=\mathcal{C}_{\xi_{\Delta, 0} \varepsilon_{\Delta}}(\alpha, 0)$ and $i$ is an imaginary unit.

Moreover, let $\bar{\xi}_{\Delta, j}(k)$ be a new virtual signal such that

$$
\bar{\xi}_{\Delta, j}(k)= \begin{cases}\xi_{\Delta, j}, & k=m p \\ 0, & \text { others }\end{cases}
$$

then the following results yield.

Corollary 5. Assume that $\bar{\xi}_{\Delta, j}(k)$ is the sequence given in (22). Then the cyclic spectral associated with $\bar{\xi}_{\Delta, j}(k)$ are given by

$$
\begin{aligned}
& \mathcal{S}_{\bar{\xi}_{\Delta, j} \varepsilon_{\Delta}}(\alpha, \omega)=\sum_{\tau=-\infty}^{\infty} \mathcal{C}_{\bar{\xi}_{\Delta, j} \varepsilon_{\Delta}}(\alpha, \tau) e^{-i \omega \tau} \\
= & e^{i j(\alpha-\omega)} \mathcal{X} \\
& \mathcal{S}_{\bar{\xi}_{\Delta, j} \xi_{\Delta, j}}(\alpha, \omega)=\sum_{\tau=-\infty}^{\infty} \mathcal{C}_{\bar{\xi}_{\Delta, j} \xi_{\Delta, j}}(\alpha, \tau) e^{-i \omega \tau} \\
= & \left(1+e^{-i(\alpha-\omega)}+\cdots+e^{-i(p-1)(\alpha-\omega)}\right) \mathcal{X} \\
& \mathcal{S}_{\bar{\xi}_{\Delta, j} \bar{\xi}_{\Delta, j}}(\alpha, \omega)=\sum_{\tau=-\infty}^{\infty} \mathcal{C}_{\bar{\xi}_{\Delta, j} \bar{\xi}_{\Delta, j}}(\alpha, \tau) e^{-i \omega \tau} \\
= & \mathcal{X}
\end{aligned}
$$

Furthermore the model in (12) leads to the cyclic spectral of $w_{\Delta}(k)$ and $e_{\Delta}(k)$ in following Lemma.

Lemma 6. Let the sequence of $w_{\Delta}(k)$ be given in (14). Then the cyclic spectral of $\mathcal{S}_{w_{\Delta} w_{\Delta}}(\alpha, \omega)$ and the cross cyclic spectral $\mathcal{S}_{w_{\Delta} e_{\Delta}}(\alpha, \omega)$ can be expressed as follows.

$$
\begin{aligned}
& \mathcal{S}_{w_{\Delta} w_{\Delta}}(\alpha, \omega) \\
= & \sum_{j=0}^{p-1} \frac{F_{j}\left(e^{-i p \omega}\right) F_{j}\left(e^{-i p(\alpha-\omega)}\right)}{E\left(e^{-i p \omega}\right) E\left(e^{-i p(\alpha-\omega)}\right)} \mathcal{S}_{\xi_{\Delta, j} \xi_{\Delta, j}}(\alpha, \omega)
\end{aligned}
$$

$$
\mathcal{S}_{w_{\Delta} e_{\Delta}}(\alpha, \omega)
$$




$$
\begin{aligned}
= & \sum_{j=0}^{p-1} \frac{F_{j}\left(e^{-i p \omega}\right)}{E\left(e^{-i p \omega}\right)} H_{\Delta}\left(e^{-i(\alpha-\omega)}\right) \mathcal{S}_{\xi_{\Delta, j} \varepsilon_{\Delta}}(\alpha, \omega) \\
= & H_{\Delta}\left(e^{i(\alpha-\omega)}\right) H_{\Delta}\left(e^{-i(\alpha-\omega)}\right) \\
& \quad\left(1+e^{-i \omega}+\cdots+e^{-i(p-1) \omega}\right) \mathcal{X}
\end{aligned}
$$

Similarly as $\bar{\xi}_{\Delta, j}$, let $\bar{w}_{\Delta}(k)$ be

$$
\bar{w}_{\Delta}(k)= \begin{cases}w(m)=e(m), & k=m p \\ 0, & \text { others }\end{cases}
$$

then we also have the following result.

Corollary 7. The following relation of cyclic spectral associated with $w_{\Delta}(k)$ and $\bar{w}_{\Delta}(k)$ holds.

$$
\begin{gathered}
\mathcal{S}_{w_{\Delta}, w_{\Delta}}(\alpha, \omega) \\
=\left(1+e^{-i \omega}+\cdots+e^{-i(p-1) \omega}\right) \mathcal{S}_{\bar{w}_{\Delta} w_{\Delta}}(\alpha, \omega) \\
=\left(1+e^{-i \omega}+\cdots+e^{-i(p-1) \omega}\right)\left(1+e^{-i(\alpha-\omega)}\right. \\
\left.+\cdots+e^{-i(p-1)(\alpha-\omega)}\right) \mathcal{S}_{\bar{w}_{\Delta} \bar{w}_{\Delta}}(\alpha, \omega) \\
\mathcal{S}_{w_{\Delta}, e_{\Delta}}(\alpha \cdot \omega)=\left(1+e^{-i \omega}+\cdots+e^{-i(p-1) \omega}\right) \\
\mathcal{S}_{\bar{w}_{\Delta} e_{\Delta}}(\alpha, \omega)
\end{gathered}
$$

On the other hand, following the framework in (Tontiruttananon and Tugnait, 1998; Giannakis, 1995), the cyclic spectral of the plant input-output in the output inter-sampling approach are given in Theorem 8.

Theorem 8. The control input associated cyclic spectral $\mathcal{S}_{\bar{u}_{\Delta}, \bar{u}_{\Delta}}(\alpha, \omega)$, the cross cyclic spectral of control input and plant output $\mathcal{S}_{\bar{u}_{\Delta}, y_{\Delta}}(\alpha, \omega)$ and cross cyclic spectral of control input and noise $\mathcal{S}_{\bar{u}_{\Delta,}, \bar{e}_{\Delta}}(\alpha, \omega)$ are given as follows respectively.

$$
\begin{gathered}
\mathcal{S}_{\bar{u}_{\Delta}, \bar{u}_{\Delta}}(\alpha, \omega)=\frac{-K\left(e^{-i p \omega}\right)}{1+K\left(e^{-i p \omega}\right) G\left(e^{-i p \omega}\right)} \times \\
\frac{-K\left(e^{-i p(\alpha-\omega)}\right)}{1+K\left(e^{-i p(\alpha-\omega)}\right) K\left(e^{-i p(\alpha-\omega)}\right)} \mathcal{S}_{\bar{w}_{\Delta}, \bar{w}_{\Delta}}(\alpha, \omega) \\
\mathcal{S}_{\bar{u}_{\Delta, e_{\Delta}}}(\alpha, \omega)=\frac{-K\left(e^{-i p \omega}\right)}{1+K\left(e^{-i p \omega}\right) G\left(e^{-i p \omega}\right)} \times \\
\mathcal{S}_{\bar{w}_{\Delta}, e_{\Delta}}(\alpha, \omega) \\
\mathcal{S}_{\bar{u}_{\Delta}, y_{\Delta}}(\alpha, \omega)=G_{\Delta}\left(e^{-i(\alpha-\omega)}\right) \mathcal{S}_{\bar{u}_{\Delta}, \bar{u}_{\Delta}}(\alpha, \omega) \\
\left(1+e^{-i(\alpha-\omega)}+\cdots+e^{-i(p-1)(\alpha-\omega)}\right) \\
+\mathcal{S}_{\bar{u}_{\Delta, e_{\Delta}}}(\alpha, \omega)
\end{gathered}
$$

where

$$
\bar{u}_{\Delta}(k)= \begin{cases}u(m), & k=m p \\ 0, & \text { others }\end{cases}
$$

Furthermore, the cyclic spectral of $\bar{u}_{\Delta}(k)$ and $y_{\Delta}(k)$ contain the following information.

Theorem 9. Let $\Omega(\alpha, \omega)$ be a frequency transfer function given by

$$
\Omega(\alpha, \omega)=\frac{\mathcal{S}_{\bar{u}_{\Delta, y_{\Delta}}}(\alpha, \omega)}{\mathcal{S}_{\bar{u}_{\Delta}, \bar{u}_{\Delta}}(\alpha, \omega)}
$$

Then the denominator of $\Omega(\alpha, \omega)$ is $A\left(e^{i p \omega}\right) D\left(e^{i p \omega}\right)$ $\sum_{j=0}^{p-1} F_{j}\left(e^{-i p \omega}\right) F_{j}\left(e^{-i p(\alpha-\omega)}\right)$.

Under the special case where $e_{\Delta}(k)$ is an i.i.d white noise, i.e., $H_{\Delta}\left(q^{-1}\right)=1$, the correlation cyclic spectral of $\mathcal{S}_{u_{\Delta} e_{\Delta}}(\alpha, \omega)$ holds the following property.

Corollary 10. When $e_{\Delta}(k)$ is an i.i.d white noise, $\mathcal{S}_{u_{\Delta} e_{\Delta}}\left(\alpha_{1}, \omega\right)=\mathcal{S}_{u_{\Delta} e_{\Delta}}\left(\alpha_{2}, \omega\right)$ and $\mathcal{S}_{\bar{u}_{\Delta} e_{\Delta}}\left(\alpha_{1}, \omega\right)=$ $\mathcal{S}_{\bar{u}_{\Delta} e_{\Delta}}\left(\alpha_{2}, \omega\right)$ hold.

\subsection{Estimation of Cyclic Spectral}

The cyclic spectral can be estimated using the definitions, and the estimation variance could also be reduced by introducing an appropriate window function (Ljung, 1999). To calculate more easily, here another estimation algorithm is considered by using fast Fourier transform (FFT).

(1) Separate the observation data $u(m), y_{j}(m)$ into $L$ blocks, and each block has $N$ data samples.

(2) Perform FFT for the $l$-th data block, and denote the results as $U^{(l)}(\omega), Y_{j}^{(l)}(\omega)$.

(3) Calculate the spectral of $u(m)$ and $y_{j}(m)$ by

$$
\begin{aligned}
& \overline{U U}(\omega)=\frac{1}{L} \sum_{l=1}^{L} U^{(l)}(\omega)\left(U^{(l)}(\omega)\right)^{*}(3 \\
& \overline{U Y}_{j}(\omega)=\frac{1}{L} \sum_{l=1}^{L} U^{(l)}(\omega)\left(Y_{j}^{(l)}(\omega)\right)^{*}(35)
\end{aligned}
$$

(4) Denote the inverse Fourier transform of $\overline{U U}(\omega), \overline{U Y}_{j}(\omega)$ as $h_{u u}(k), h_{u y_{j}}(k)$ for $k=$ $0, \cdots, N-1$.

(5) The estimates of cyclic spectral are given by

$$
\begin{aligned}
& \hat{\mathcal{S}}_{\bar{u}_{\Delta}, \bar{u}_{\Delta}}(\alpha, \omega) \\
& =\mathcal{F}\{\overbrace{h_{u u}(0), \underbrace{0 \cdots 0}_{p-1}, h_{u u}(1), \underbrace{0 \cdots 0}_{p-1}, \cdots}^{p N}\} \\
& \hat{\mathcal{S}}_{\bar{u}_{\Delta}, y_{\Delta}}(\alpha, \omega) \\
& =\mathcal{F}\{\overbrace{h_{u y_{0}}(0), \underbrace{0 \cdots 0}_{p-1}, h_{u y_{0}}(1), \underbrace{0 \cdots 0}_{p-1}, \cdots}^{p N}\} \\
& +e^{i \alpha} \mathcal{F}\{\overbrace{0, h_{u y_{1}}(0), \underbrace{0 \cdots 0}_{p-1}, h_{u y_{1}}(1), \underbrace{0 \cdots 0}_{p-1}, \cdots}^{p N}\} \\
& +\cdots+e^{i(p-1) \alpha} \cdot \mathcal{F} \\
& \{\overbrace{\underbrace{0 \cdots 0}_{p-1}, h_{u y_{p-1}}(0), \underbrace{0 \cdots 0}_{p-1}, \cdots, h_{u y_{p-1}}(N-1)}^{p N}\}
\end{aligned}
$$

where $\mathcal{F}$ is Fourier transform. 


\section{IDENTIFICATION ALGORITHM}

By using the plant input $u_{\Delta}(k)$ and output $y_{\Delta}(k)$, the plant model can be estimated through the following procedures.

(1) Estimation of $\mathcal{S}_{\bar{u}_{\Delta, y_{\Delta}}}(\alpha, \omega)$ and $\mathcal{S}_{\bar{u}_{\Delta}, \bar{u}_{\Delta}}(\alpha, \omega)$ in $\omega \in[0,2 \pi)$.

(2) Estimation of denominator $A\left(e^{i p \omega}\right)$

The parametric model of $\Omega\left(e^{i p \omega}\right)$ can be obtained from (33) (Kollár, 1993). Denote the poles of $G\left(z^{-1}\right)$ as $\rho_{l}$, and the pole set as $\mathcal{P}$. If any of $1 / \rho_{l} e^{-i j \frac{2 \pi}{p}} \notin$ $\mathcal{P}$, then $A\left(e^{i p \omega}\right)$ can be separated from $A\left(e^{i p \omega}\right) D\left(e^{i p \omega}\right) \sum_{j=0}^{p-1} F_{j}\left(e^{-i p \omega}\right) F_{j}\left(e^{-i p(\alpha-\omega)}\right)$

(3) Estimation of instrumental variable $\Theta\left(e^{-i p \omega}\right)$

From $\hat{\mathcal{S}}_{\bar{u}_{\Delta}, \bar{u}_{\Delta}}(\alpha, \omega),\left(A\left(e^{-i p \omega}\right) C\left(e^{-i p \omega}\right)+\right.$ $\left.B\left(e^{-i p \omega}\right) D\left(e^{-i p \omega}\right)\right) E\left(e^{-i p \omega}\right)$ can be obtained. Using the estimate $\hat{A}\left(e^{-i p \omega}\right)$ the instrumental variable $\Theta\left(e^{-i p \omega}\right)$ can be calculated by

$\Theta\left(e^{-i p \omega}\right)=\frac{K\left(\widehat{e^{-i p \omega}}\right)}{\left(1+K\left(e^{-i p \omega}\right) G\left(e^{-i p \omega}\right)\right) E\left(e^{-i p \omega}\right)}$

(4) Estimation of denominator of noise model $E_{\Delta}\left(e^{i \omega}\right)$

It can be seen that

$$
\frac{\hat{\mathcal{S}}_{\bar{u}_{\Delta, y_{\Delta}}}(\alpha, \omega)}{\hat{\Theta}\left(e^{-i p \omega}\right)} \hat{A}\left(e^{i p \omega}\right)=\frac{\Xi(\alpha, \omega)}{E_{\Delta}\left(e^{-i(\alpha-\omega)}\right)}(39)
$$

holds, where $\Xi(\alpha, \omega)$ is given by

$$
\begin{aligned}
& \Xi(\alpha, \omega)=B_{\Delta}\left(e^{-i(\alpha-\omega)}\right) \bar{A}_{\Delta}\left(e^{-i(\alpha-\omega)}\right) \\
& \quad \sum_{l=0}^{p-1} e^{-i l(\alpha-\omega)} \hat{\mathcal{S}}_{\bar{u}_{\Delta}, \bar{u}_{\Delta}}(\alpha, \omega) E_{\Delta}\left(e^{-i(\alpha-\omega)}\right) \\
& \quad+\bar{E}_{\Delta}\left(e^{i(\alpha-\omega)}\right) F_{\Delta}\left(e^{i(\alpha-\omega)}\right) F_{\Delta}\left(e^{-i(\alpha-\omega)}\right)
\end{aligned}
$$

Then $E_{\Delta}\left(e^{-i(\alpha-\omega)}\right)$ can be estimated. So $\hat{E}\left(e^{-i p \omega}\right)$ is calculated from $\hat{E}_{\Delta}\left(e^{-i(\alpha-\omega)}\right)$.

(5) Estimation of numerator $B\left(e^{i p \omega}\right)$

Using $\hat{E}\left(e^{-i p \omega}\right)$ and instrumental variable $\Theta\left(e^{-i p \omega}\right)$ the following spectral

$$
\begin{aligned}
& \widehat{\mathcal{\mathcal { S }}}_{\bar{u}_{\Delta}, \bar{u}_{\Delta}}(\alpha, \omega)=\frac{\hat{\mathcal{S}}_{\bar{u}_{\Delta}, \bar{u}_{\Delta}}(\alpha, \omega)}{\hat{\Theta}\left(e^{-i p \omega}\right) \hat{E}\left(e^{-i p \omega}\right)} \\
& \widehat{\mathcal{\mathcal { S }}}_{\bar{u}_{\Delta, y_{\Delta}}}(\alpha, \omega)=\frac{\hat{\mathcal{S}}_{\bar{u}_{\Delta, y \Delta}}(\alpha, \omega)}{\hat{\Theta}\left(e^{-i p \omega}\right) \hat{E}\left(e^{-i p \omega}\right)}
\end{aligned}
$$

are obtained. Then from the equation

$$
\begin{aligned}
& \operatorname{Im}\left(\widehat{\overline{\mathcal{S}}}_{\bar{u}_{\Delta, y_{\Delta}}}(\alpha, \omega)\right) \\
= & \operatorname{Im}\left(B_{\Delta}\left(e^{-i(\alpha-\omega)}\right) \bar{A}_{\Delta}\left(e^{-i(\alpha-\omega)}\right)\right. \\
& \left.\sum_{l=0}^{p-1} e^{-i l(\alpha-\omega)} \frac{\widehat{\overline{\mathcal{S}}}_{\bar{u}_{\Delta} \bar{u}_{\Delta}(\alpha, \omega)}}{\left.A\left(e^{-i p(\alpha-\omega)}\right)\right)}\right)
\end{aligned}
$$

the coefficients of $B_{\Delta}\left(q^{-1}\right) \bar{A}_{\Delta}\left(q^{-1}\right)$ are estimated, so the estimates of $B\left(z^{-1}\right)$ can be obtained by using Corollary 3 .

Note that $\mathcal{S}_{\bar{u}_{\Delta, y_{\Delta}}}(\alpha, \omega)$ and $\mathcal{S}_{\bar{u}_{\Delta}, \bar{u}_{\Delta}}(\alpha, \omega)$ can be estimated when the closed-loop is stabilized by feedback controller, even though the plant is unstable. So the proposed algorithm can be applied for unstable plant identification directly.

\section{NUMERICAL EXAMPLES}

The effectiveness of the proposed algorithm is illustrated in this section through two simulation examples.

\subsection{Second-Order Plant}

Consider a second-order unstable plant, whose continuous time transfer function is given by

$$
G(s)=\frac{0.2165 s+1.9646}{s^{2}+0.6444 s-0.4257}
$$

Provide that the holding period of the controller is $T=1.0 \mathrm{~s}$. And the controller is a proportional controller given by

$$
K\left(z^{-1}\right)=0.35
$$

The output inter-sampling rate is chosen as $p=2$, i.e., the output sampling interval is $\Delta=T / 2=$ $0.5 \mathrm{~s}$. Then the T-model and the $\Delta$-model of the plant are given by

$$
\begin{aligned}
& G\left(z^{-1}\right)=\frac{z^{-1}+0.5 z^{-2}}{1-1.85 z^{-1}+0.525 z^{-2}} \\
& G_{\Delta}\left(q^{-1}\right)=\frac{0.3173 q^{-1}+0.1063 q^{-2}}{1+1.863 q^{-1}+0.7246 q^{-2}}
\end{aligned}
$$

respectively. It can be seen that the poles of $T$ model are 1.5, 0.35. On the other hand, assume that the noise $e_{\Delta}(k)$ is a white noise with zero mean and variance 1.0, and the reference signal $r=0$. The plant input and output signals are collected for $8192 T$. From the PE property of the reference signal $r$ and the controller structure, it can be seen that the conventional identifiability is not fulfilled, therefore the plant cannot be identified by using conventional methods.

For comparison both the proposed algorithm and the revised prediction error method (RPEM) given in (Forssell and Ljung, 1999) are used. The cyclic spectral of $\mathcal{S}_{\bar{u}_{\Delta, y_{\Delta}}}(0, \omega)$ is plotted in Fig 3 . The simulation is performed for 10 trials and the estimation results are summarized in Table 1.
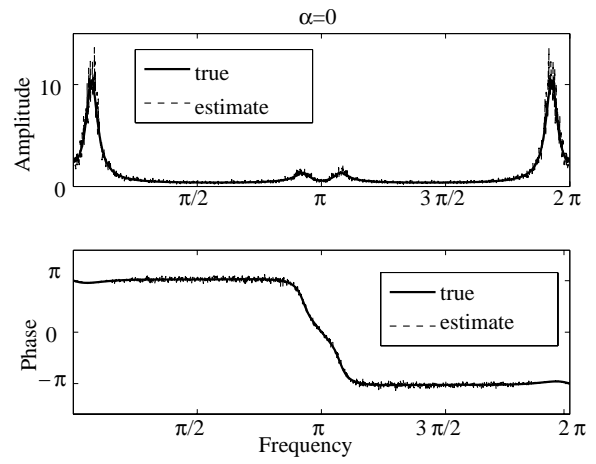

Fig. 3. Estimation of cyclic spectral $\mathcal{S}_{\bar{u}_{\Delta}, y_{\Delta}}(0, \omega)$ 
From Table 1, it is seen that both the proposed algorithm and RPEM can estimate the unstable pole of plant model in (46) or (47). The proposed one uses the spectral estimates in frequency domain thus the estimation variance is larger than that in time domain by RPEM algorithm.

Table 1. Estimation results (10 trials)

\begin{tabular}{crrrr}
\hline & $a_{1}$ & $a_{2}$ & $b_{1}$ & $b_{2}$ \\
\hline True & -1.8500 & 0.5250 & 1.0000 & 0.5000 \\
\hline Proposed & -1.8503 & 0.5382 & 1.0156 & 0.4751 \\
algorithm & \pm 0.019 & \pm 0.015 & \pm 0.060 & \pm 0.055 \\
\hline Revised & -1.8491 & 0.5235 & 1.000 & 0.5030 \\
PEM & \pm 0.003 & \pm 0.005 & \pm 0.005 & \pm 0.011 \\
\hline
\end{tabular}

\subsection{3rd-Order Plant}

The continuous time transfer function of the plant model is given as follows.

$$
G(s)=\frac{-2.8851 \times 10^{5}}{s^{3}+118.72 s^{2}-1483.9 s-99275}(48)
$$

The holding period of the controller is $T=0.002 \mathrm{~s}$. The controller here is a digital PID controller whose transfer function is

$$
K\left(z^{-1}\right)=\frac{12.09+23.616 z^{-1}-11.53 z^{-2}}{1-1.4 z^{-1}+0.4 z^{-2}}
$$

The output sampling interval $\Delta$ is $\Delta=T / 2=$ 0.001 . The $T$-model and $\Delta$-model of the plant are

$$
\begin{aligned}
& G\left(z^{-1}\right) \\
= & \frac{-10^{-4}\left(0.363 z^{-1}+1.37 z^{-2}+0.322 z^{-3}\right)}{1-2.794 z^{-1}+2.582 z^{-2}-0.789 z^{-3}} \\
& G_{\Delta}\left(q^{-1}\right) \\
= & \frac{-10^{-5}\left(0.467 q^{-1}+1.812 q^{-2}+0.44 q^{-3}\right)}{1-2.89 q^{-1}+2.778 q^{-2}-0.888 q^{-3}}
\end{aligned}
$$

respectively. It can be verified easily that the $T$ model has poles 1.0643, 0.95 and 0.78 . The noise model is given by

$$
\begin{gathered}
e_{\Delta}(k)=w_{\Delta}(k)-0.547 w_{\Delta}(k-1) \\
+0.667 w_{\Delta}(k-2)
\end{gathered}
$$

where $w_{\Delta}(k)$ is a white noise with zero mean and variance 1.0. The reference signal $r$ is chosen as 0.5 . The estimated poles in 10 simulations by the proposed algorithm are summarized in Table 2, which shows that the unstable pole in model (50) are well estimated. On the other hand, the illconditioned problem occurs in the nonlinear optimization when using RPEM algorithm because there are two poles close to the unit circle. As a result, there are 6 simulations where the estimation diverges or converges to local solution within 10 simulation trials.

\section{CONCLUSIONS}

It is clarified that the plant signals have cyclic spectrum when the plant output is sampled at a
Table 2. Estimated poles (10 trials)

\begin{tabular}{cccc}
\hline & Pole 1 & Pole 2 & Pole 3 \\
\hline True & 1.0643 & 0.9500 & 0.7800 \\
\hline Proposed & 1.0640 & 0.9480 & 0.7828 \\
algorithm & \pm 0.0052 & \pm 0.0079 & \pm 0.0105 \\
\hline Revised & -0.1929 & $0.949+0.044 i$ & $0.936-0.044 i$ \\
PEM & \pm 0.9948 & $\pm(0.02+0.02 i)$ & $\pm(0.01+0.02 i)$ \\
\hline
\end{tabular}

higher rate than that of the control input, and the frequency transfer function of the inter-sampled model is also deduced. Based on the cyclic spectrum of plant output and control input, a new direct closed-loop identification algorithm in frequency domain is proposed. When the plant is stabilized by the feedback controller, the spectral of plant input and output can be calculated even though the plant is unstable; the proposed algorithm can also be used in unstable plant estimation problems directly. Furthermore, the effectiveness of the proposed method is demonstrated through numerical simulations.

\section{REFERENCES}

den Hof, P.M. J. Van, R.J.P. Schrama, R.A. de Callafon and O. H. Bosga (1995). Identification of normalized coprime factors from closed-loop experimental data. Euro. J. Control 1, 1751-1770.

Forssell, U. and L. Ljung (1999). Closed-loop identification revisited. Automatica 35, 12151241.

Giannakis, G.B. (1995). Polyspectral and cyclostationary approaches for identification of closed-loop systems. IEEE Trans. Automatic Control 40(5), 882-885.

Hansen, F.R. and G.F. Franklin (1988). On a fractional representation approach to closedloop experimental design. In: Proc. $A C C^{\prime} 88$. pp. 1319-1320. Atlanta,USA.

Kollár, I. (1993). On frequency domain identification of linear systems. IEEE Trans. on Instrumentation and Measurement 42(1), 2-6.

Ljung, L. (1999). System Identification - Theory for the User. Prentice Hall, Englewood Cliffs, N.J.

Ljung, L., I. Gustavsson and T. Söderström (1974). Identification of linear multivariable systems operating under linear feedback control. IEEE Trans. on Aut. Control AC19(6), 836-840.

Sun, L., H. Ohmori and A. Sano (1999). Direct closed-loop identification of magnetic suspension system. In: Proc. of IEEE CCA 1999. pp. 749-754. Hawaii, USA.

Sun, L., H. Ohmori and A. Sano (2001). Output inter-sampling approach to closed-loop identification. IEEE Trans. Automatic Control 46(12), 1936-1941.

Tontiruttananon, C. and J.K. Tugnait (1998). Parametric identification of closed-loop linear systems using cyclic-spectral analysis. In: American Control Conference. pp. 35973601.

Wellstead, P.E. and J. M. Edmunds (1975). Leastsquares identification of closed-loop systems. Int. J. Control 21(4), 689-699. 